\title{
Biomass Derived Reduced Graphene oxide from Abelmoschus esculentus and Cucumis melo peels as electrode material for High Performance Supercapacitors
}

\author{
${ }^{1}$ T. Kalaivani, ${ }^{2}$ S. Tharani, ${ }^{3}$ A. Prithiba \\ ${ }^{1}$ Research Scholar, ${ }^{2}$ Research Scholar, ${ }^{3}$ Asst Professor (SS) \\ Department of Chemistry, Avinashilingam Institute for Home Science and Higher Education for Women, Coimbatore.
}

\begin{abstract}
Supercapacitors, an electrochemical energy storage device has been used as one of the powerful and advanced energy storage system. For these supercapacitors, Electrode material from plant source have largely been tried as carbon source due to their eco- friendly, economic nature, large surface area and outstanding electrochemical performance. In the Present research work, Reduced Graphene oxide have been synthesized by using Abelmoschus esculentus and Cucumis melo waste peels through Hydrothermal Carbonization method and reduced in eco- friendly manner by using Modified Hummer's method. The physical and chemical properties of the produced Reduced Graphene oxide were characterized using FT-IR spectroscopy, X- Ray Diffraction, FE-SEM, Energy Dispersive Analysis of X-Ray, Thermogravimetric Analysis. Capacitance behavior of the produced Reduced Graphene oxide of Abelmoschus esculentus and Cucumis melo waste peels are examined using Electrochemical analysis. Specific capacitance of Reduced Graphene oxide of Abelmoschus esculentus and Cucumis melo were found to be $149 \mathrm{~F} / \mathrm{g}$ and $151 \mathrm{~F} / \mathrm{g}$ respectively at a current density of $2 \mathrm{~A} / \mathrm{g}$.
\end{abstract}

Keywords: Abelmoschus esculentus, Cucumis melo, Carbon Aerogels, Supercapacitors, Electrochemical properties

\section{INTRODUCTION}

Energy is one of the critical inputs for commercial growth of any Country. The need of the hour is the sustainable utilization of renewable sources to overcome the present energy scenario problems. The disproportionate amount of energy consumption in recent years has accelerated the depletion of non-renewable forcing us to rely just on renewable sources. Biomass presently affords around $10 \%$ of the world's predominant energy provisions, mostly utilized wood as fuel or charcoal for heating system and cooking purposes in economically emerging countries. Amongst the various renewable energy bases, biomass plays a dynamic role particularly in rural areas. The evolution of energy storage systems with high charge and energy densities considered as one of the potential solutions to solve global concerns [21] of fossil fuel diminution and ecological deterioration. Among several innovative electrochemical storage devices, supercapacitors concerned incredible interest as energy storage devices because of their high energy density and extensive cycle life than other energy storage devices such as capacitors, battery, fuel cell etc... [1]. Supercapacitors with carbon- based materials accomplished the electrostatic separation between electrode with high surface area and electrolyte ions. This consequence the energy accumulated in supercapacitors was linearly equivalent to the capacitance of the electrodes which makes the carbon- based materials critical. The consumption of plant materials for the production of porous carbon develops the attention of extensive obtainability and energy/green concern are considered. Among the various techniques for the preparation of carbon- based materials, carbonization is a novel technique in which porous carbon was synthesised from the plant materials and further taken for chemical or physical activation process. Among, various methods were involved for graphene preparation, the chemical reducing method is one of the prominent methods for mass construction of graphene. On the other hand, biomass derived reducing agents including orange peels [2], aloe vera [3], wild carrot root [4], Hibiscus sabdariffa.L [5], tea solution [6] [7], ginseng[8] and black soybeans[9] were reported as reduce graphene oxide. Supercapacitors are also called ultra-capacitors with a great capacitance; they have combined characteristics of batteries and capacitors in a single device. Supercapacitors are basically classified into the electric double-layer capacitors, Pseudo capacitors and Hybrid capacitors.

"Electric-double-layer capacitors (EDLC) have capacitance from the electrostatic charge accumulation at the electrode- 


\title{
International Advanced Research Journal in Science, Engineering and Technology
}

\author{
Vol. 8, Issue 8, August 2021
}

DOI: $10.17148 /$ IARJSET.2021.8852

electrolyte interface. In EDLC, the electrolyte concentration remains constant during the charging and discharging processes. In this way, energy is stored in the double- layer interface.

Pseudo capacitors- capacitance from fast and reversible redox processes at the electroactive material surface. When a potential is applied pseudo capacitors, fast and reversible faradaic reactions (redox reactions) take place on the electrode materials and involved the passage of charge across the double layer, similar to the charging and discharging processes that occur in energy storage devices, resulting in faradaic current passing through the supercapacitor cell.

Hybrid capacitors- attributed to the electrostatic double layer capacitance and Pseudo capacitance conventional capacitors stores energy by moving electrons from one electrode to another" [29].

Cucumis melo is a common fruit and it is commonly called Musk melon. The peels of this fruit are always treated as waste thrown in trash. Abelmoschus esculentus is commonly called okra or Lady's finger, here we utilize these biomasses as precursors to synthesize reduced graphene oxide for high performing supercapacitors. The Cucumis melo derived carbon materials shows very high capacitance than the carbon materials derived from Abelmoschus esculentus.

\subsection{Materials}

\section{EXPERIMENTAL SECTIONS}

Chemicals such as graphite powder, $\mathrm{KMnO}_{4}$ (potassium permanganate), $\mathrm{H}_{2} \mathrm{SO}_{4}$ (sulphuric acid) and $\mathrm{H}_{2} \mathrm{O}_{2}$ (hydrogen peroxide) were used in this study. Abelmoschus esculentus vegetable peels and Cucumis melo fruit peels were collected from a nearby farm in the Coimbatore region. They were authenticated by the Botany department of our university and a voucher specimen was deposited.

\subsection{Synthesis of Graphene oxide}

Graphene oxide was prepared by adopting the modified Hummer's method. $10 \mathrm{~g}$ of Graphite was taken in a $500 \mathrm{ml}$ flask. Added $300 \mathrm{ml}$ of concentrated sulphuric acid and the mixture were slowly stirred in an ice bath for 10 minutes. $20 \mathrm{~g}$ of $\mathrm{KMnO}_{4}$ (potassium permanganate) was added over for 1 hour. The reaction mixture was continuously stirred for 4 hours along with the ice bath. To it added $150 \mathrm{ml}$ of $5 \% \mathrm{H}_{2} \mathrm{O}_{2}$ gradually for 30 minutes. The mixture was filtered and the precipitate was wash away with distilled water until the $\mathrm{pH}$ of filtrate is increased to 6-7. The obtained precipitate was oven dried for 24 hours at $80^{\circ} \mathrm{C}$. The black precipitate obtained was made into minute powder for further studies [20].

\subsection{Preparation of plant Extract}

The waste peels of Abelmoschus esculentus [AE] and Cucumis melo [CM]were carefully selected and 50g of these peels were immersed in deionised water $(200 \mathrm{ml})$ and refluxed for 4 hours. Then the obtained mixture was filtered and the filtrate was stored at $0-4^{\circ} \mathrm{C}$. [10]

\subsection{Synthesis of reduced graphene oxide}

$200 \mathrm{mg}$ of above prepared $\mathrm{GO}$ was distributed in $400 \mathrm{ml}$ of deionized water and taken for sonication for 30 minutes to get $\mathrm{GO}$ sheets. The mixture was added to $85 \mathrm{ml}$ of $\mathrm{AE}$ and $\mathrm{CM}$ extract taken in the round bottom flask and stirred for 24 hours at room temperature. After the filtration, black powder was collected, which was washed with deionised water to get rid of the excess plant residue and taken for sonication. This suspension was taken for centrifuge at $4000 \mathrm{rpm}$ for 30 minutes. After that, the supernatant was removed and the black precipitate was dried in vacuum at $80^{\circ} \mathrm{C}$ [2]. The highly reduced graphene from Abelmoschus esculentus [AE] and Cucumis melo [CM] was denoted as AERGO and CMRGO respectively.

\subsection{Electrode fabrication}

The mixture of active material, conductive material (graphite) and a binder (polyvinyl chloride) taken in the ratio of 85:10:5 to form homogeneous slurry for the preparation of electrode. The slurry was compelled between two pieces of aluminium under $300 \mathrm{~kg} \mathrm{~cm}^{2}$. After the vacuum-drying at $100^{\circ} \mathrm{C}$ for overnight, the electrodes were impregnated with 30 wt.\% $1 \mathrm{M} \mathrm{H}_{2} \mathrm{SO}_{4}$ electrolyte under vacuum to guarantee thorough wetting. The experiments were carried out in a classical three-electrode electrochemical cell. Biologic system interfaced with an IBM computer and EC lab 10.4 software was used for data acquisition and analysis [24]. Working electrode- active material coated electrode, counter electrode - Pt electrode (platinum), reference electrode- calomel electrode.

\subsection{Characterisation}

Fourier Transform infrared spectroscopy (FT-IR) was carried out over the wave number range of 4500 to $400 \mathrm{~cm}^{-1}$ using Perkin Elmer FT-IR spectrophotometer with the SOFTWARE - OPUS version 6.5. Scanning electron microscopy (SEM) JEOL MODEL JSM 6360 was used to examine the morphology of reduced graphene oxide. The thermal stability 


\section{International Advanced Research Journal in Science, Engineering and Technology}

Vol. 8, Issue 8, August 2021

DOI: $10.17148 /$ IARJSET.2021.8852

measurements were performed using Exstar- SII-TG/DTA 6300. The Diffraction patterns were analysed using XPERT PRO. The particle diameter distributions estimated with a dynamic light scattering analyser (DLS) (LA-910, HORIBA). The analyses performed at $25^{\circ} \mathrm{C}$ with a $580 \mathrm{~nm}$ laser at a scattering angle of $90^{\circ}$.

\subsection{FT-IR spectroscopy}

\section{RESULTS AND DISCUSSIONS}

The FT-IR spectroscopy is taken to recognize the functional groups attached to the surface of biomass. It also determined the changes occurred during processing of biomass. The peaks at around $2981 \mathrm{~cm}^{-1}$ corresponded to stretching vibrations of aliphatic $\mathrm{C}-\mathrm{H}$ and the peak at $1056-1273 \mathrm{~cm}^{-1}$ were indicated to $\mathrm{C}-\mathrm{O}$ stretching vibrations in hydroxyl group, ester or ether groups and $3441 \mathrm{~cm}^{-1} \mathrm{O}-\mathrm{H}$ stretching vibrations. Remarkably, the intensities of the peaks may be attributed to the hydroxyl groups (3000-3700 and 1000-1450 $\mathrm{cm}^{-1}$ ) showed weak when compared with GO and the peak $\mathrm{C}=\mathrm{O}$ groups seemed at $1759 \mathrm{~cm}^{-1}$, which was attributed to the dehydration reactions. Meanwhile, the new vibration peaks at 1639 and $1523 \mathrm{~cm}^{-1}$ unambiguously showed the presence of benzene structure in the samples and the bands at $844-740 \mathrm{~cm}^{-1} \mathrm{were}$ assigned to aromatic C-H out-of-plane bending vibrations [26].

The functional groups of the obtained CMRGO were identified by (FT-IR) Spectroscopy as shown in (Fig 1: b). The broad and strong band at $3741 \mathrm{~cm}^{-1}$ is assigned to the $\mathrm{OH}$ Stretching and the peaks around at 1693 and $1647 \mathrm{~cm}^{-1}$ indicates the $\mathrm{C}=\mathrm{O}$ and $\mathrm{C}=\mathrm{C}$ stretching vibrations respectively. The peaks at near $1462 \mathrm{~cm}^{-1}$ are assigned to either carboxylic $\mathrm{O}-\mathrm{H}$

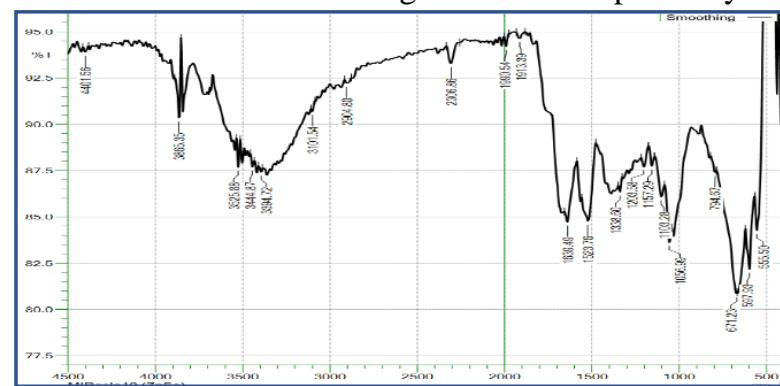

Figure 1.a: FT-IR Spectral peaks of AERGO

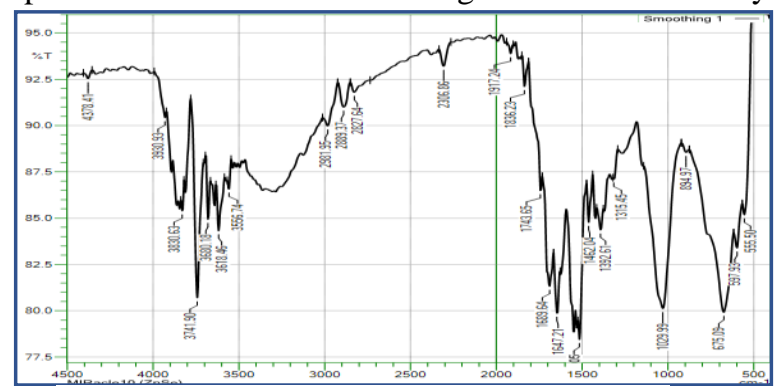

Figure $1 . b$ :FT-IR Spectral peaks of CMRGO

deformation or $\mathrm{C}-\mathrm{H}$ bending vibration. The peaks at $1026 \mathrm{~cm}^{-1}$ are assigned to the $\mathrm{C}-\mathrm{O}-\mathrm{C}$ stretching vibration and deformation vibration of vinyl O-H respectively [13]. This indicated that AE and CM peel extracts successfully reduced the GO samples.

\begin{tabular}{|l|l|l|}
\hline \multicolumn{2}{|c|}{ Frequency $\left(\mathbf{c m}^{-1}\right)$} \\
\hline AERGO & CMRGO & Assignment \\
\hline 3498 & 3460 & OH stretch \\
2875 & 2765 & C-H stretch \\
2306 & 2380 & -C $\equiv$ N stretch \\
- & 1689 & C=O stretch, C-C in ring aromatics \\
1550 & 1512 & C-H bend \\
1033 & 1091 & C-O-C stretch \\
1286 & 1226 & C-O stretch \\
- & 1098 & C-C stretch \\
675 & 686 & O-H bend \\
\hline
\end{tabular}

\section{Table:1 FT-IR spectral values of AERGO and CMRGO}

\subsection{Thermogravimetric analysis}

The AERGO and CMRGO was seen to be unstable and decompose below $100^{\circ} \mathrm{C}$ may be due to the existence of moisture and water. Severe reduction was seen to occur at $190^{\circ} \mathrm{C}$. This may be due to removal of unstable oxygenated groups which yields $\mathrm{CO}, \mathrm{CO}_{2}$ and steam. In Graphene oxide, a slighter exothermic peak is seen around $212^{\circ} \mathrm{C}$ representing the release of $\mathrm{CO}$ and $\mathrm{CO}_{2}$ from $\mathrm{GO}$ triggered by the demolition of oxygen containing functional groups. The GO shows the similar results as AERGO and CMRGO for below $190^{\circ} \mathrm{C}$ [27]. 

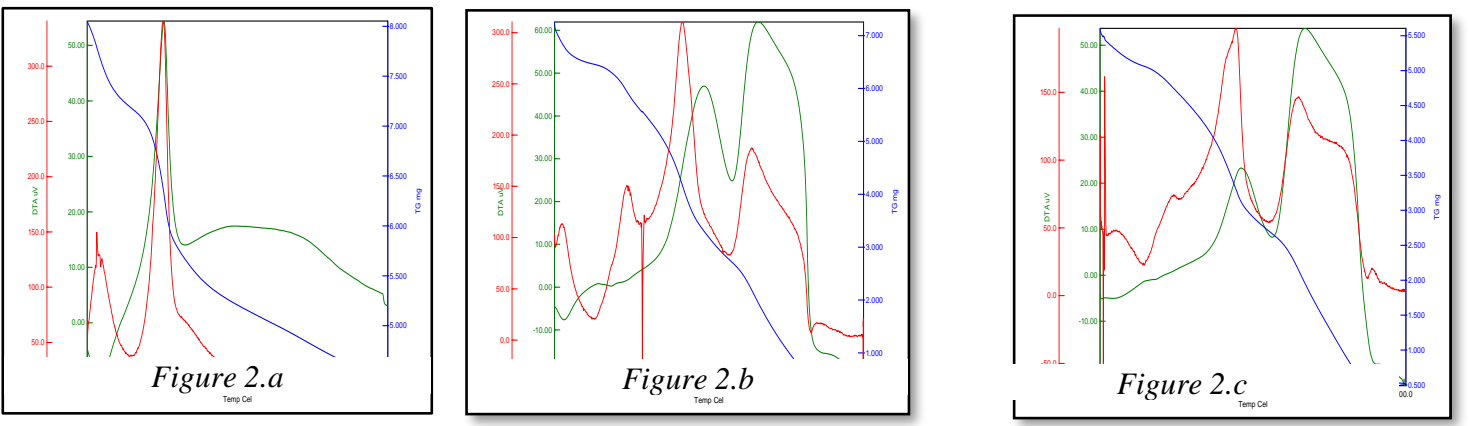

Fig: 2.a, 2.b and 2.c: TGA plot of GO, AERGO and CMRGO

\subsection{Scanning electron microscopy}

The surface morphology of the prepared reduced graphene oxide was evaluated by scanning electron microscopy. The SEM images of AERGO and CMRGO (Figure :3,4) exhibits a continuous, loose but distorted layered microstructure. Such a typical construction can simplify the subsequent $\mathrm{KOH}$ impregnation and activation. The figures were seen to be irregular in shape and tend to show agglomeration phenomena in varying degrees. It was clear seen that small macropores are present [28].
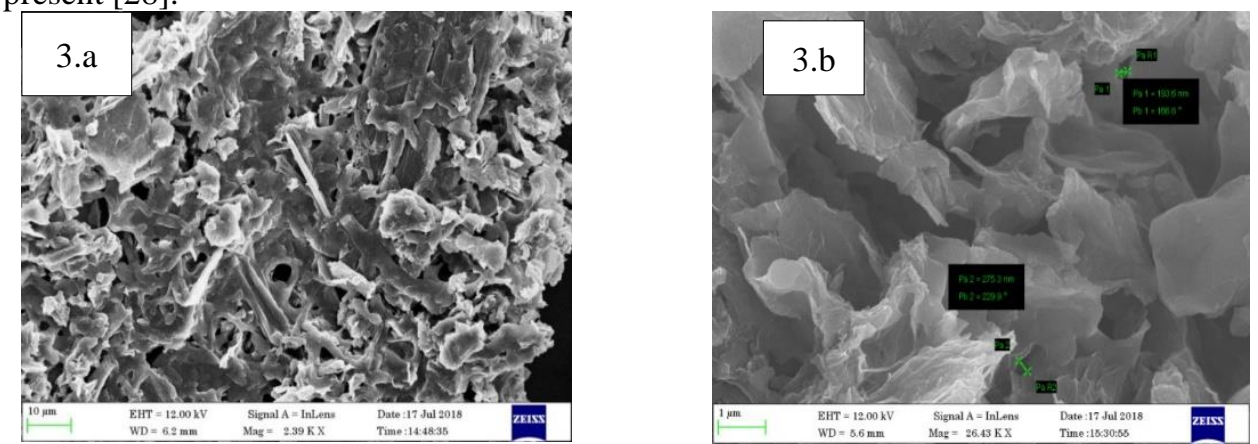

Fig: 3 SEM images of a) AERGO b) Pore size of AERGO by SEM image
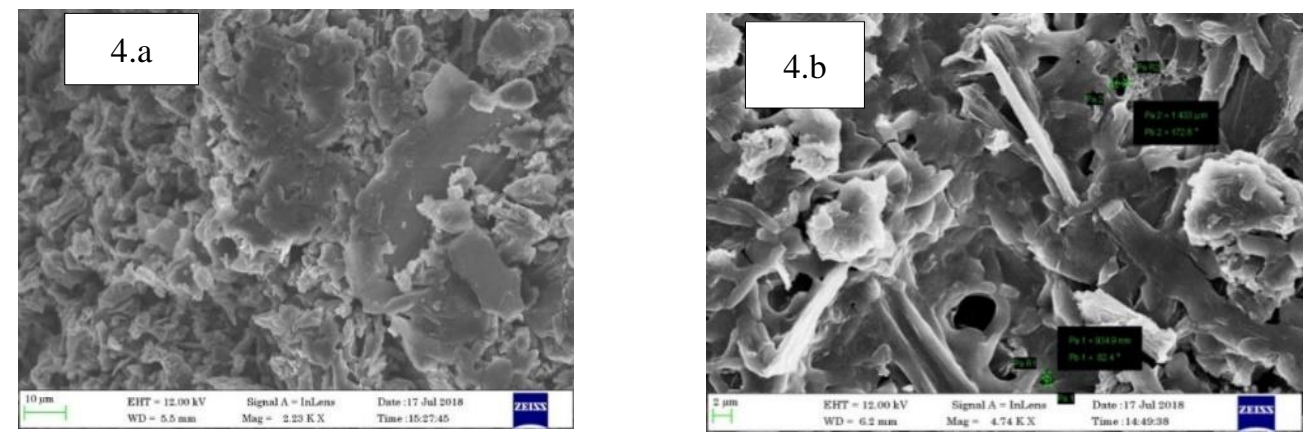

Fig 4: a) SEM images of CMRGO b) Pore size of CMRGO by SEM images

\subsection{EDX Studies}

EDX spectra were taken for the determination of elements present on the surface of AERGO, CMRGO (Figure 5.a, 5.b). Elemental analysis of AERGO indicated very low oxygen content which might be due to increase in gasification reactions [14]. Thus, it is concluded that the composition of carbon and oxygen in the prepared reduced graphene oxide shows good agreement with FT- IR results. 
Vol. 8, Issue 8, August 2021

DOI: $10.17148 / I A R J S E T .2021 .8852$

\begin{tabular}{|l|l|l|l|l|}
\hline \multirow{2}{*}{ Element } & \multicolumn{2}{|c|}{ AERGO } & \multicolumn{2}{c|}{ CMRGO } \\
\cline { 2 - 5 } & Wt \% & At \% & Wt \% & At \% \\
\hline $\mathrm{C}$ & 89.32 & 84.55 & 52.11 & 59.44 \\
$\mathrm{O}$ & 6.85 & 5.28 & 47.01 & 40.25 \\
$\mathrm{Na}$ & - & -- & - & - \\
$\mathrm{Mg}$ & - & -- & - & - \\
$\mathrm{Al}$ & - & -- & - & - \\
$\mathrm{Si}$ & - & -- & - & - \\
$\mathrm{Cl}$ & - & -- & - & - \\
$\mathrm{K}$ & - & 0.08 & 0.45 & 0.16 \\
$\mathrm{Ca}$ & 2.27 & 0.07 & 0.30 & 0.10 \\
$\mathrm{Cu}$ & 2.22 & 0.05 & - & - \\
$\mathrm{S}$ & 0.28 & - & 0.13 & 0.06 \\
& - & & & \\
\hline
\end{tabular}

Table.2: EDX Analysis of AERGO

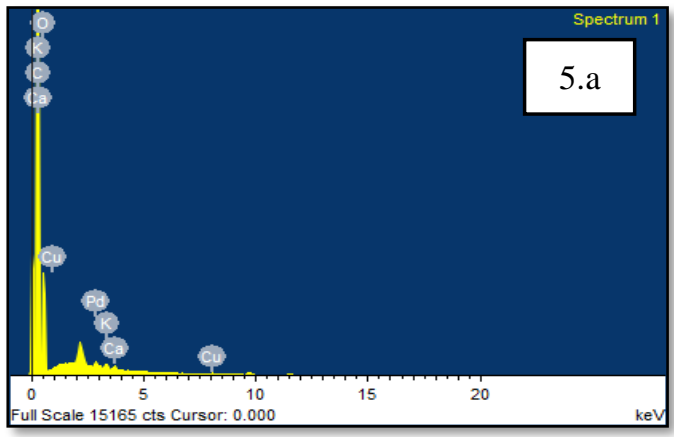

Fig: 5.a: EDX image of AERGO

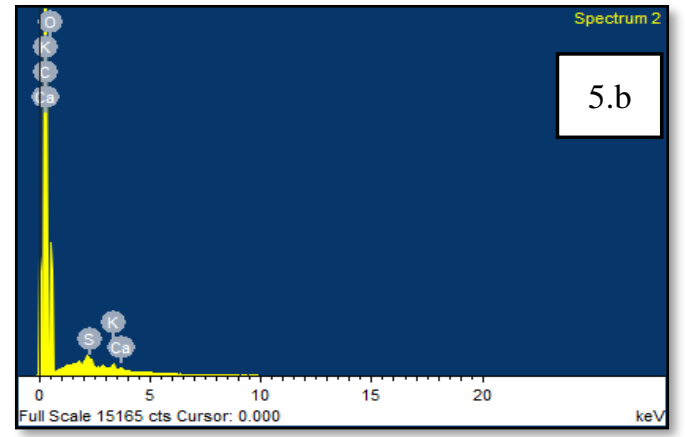

Fig: 5.b: EDX image of CMRGO

\subsection{XRD Analysis}

X-Ray Diffraction (XRD) is used to determine the nature of the materials. The XRD pattern of GO showed at $2 \theta=10.7^{\circ}$, which corresponds to d-spacing of $0.82 \mathrm{~nm}$. Figure 6 showed the XRD analysis of AERGO and CMRGO showed two apparent broad diffraction peaks (around at $28.0^{\circ}$ to 44.0 ) may be attributed to the arranged carbon sheet structure (002) and well-ordered graphite structure (100). In addition, a rise in the intensity of the low-angle region reveals a high porosity in the sample. From the diffractograms, two broad humps at about $29^{\circ}$ and $41^{\circ}$ can be observed which attributed to the graphene-like domains [23]. By using a diffraction peak of $2 \theta=43^{\circ}(\mathrm{AERGO}) ; 42^{\circ}(\mathrm{CMRGO})$,

"The crystallite size was calculated from Scherrer's equation, $L=K \lambda / \beta \cos \theta$ where $L$ is a crystallite size, $\lambda$ is wavelength of $\mathrm{X}$-ray, $\beta$ is half width, $\mathrm{K}$ is a constant (0.1542 in this run)" [11].

The crystallite size was found to be $19 \mathrm{~nm}$ and $19 \mathrm{~nm}$ for AERGO and CMRGO.

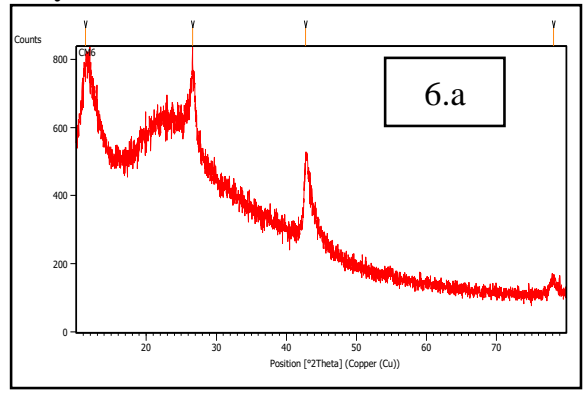

Fig: 6.a XRD pattern of AERGO

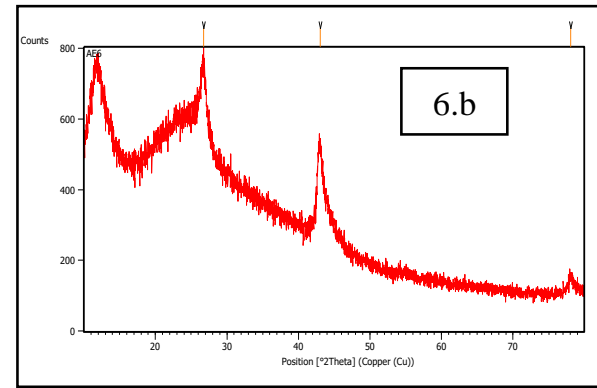

Fig: 6.b XRD pattern of CMRGO 
Vol. 8, Issue 8, August 2021

DOI: $10.17148 / I A R J S E T .2021 .8852$

\begin{tabular}{|c|c|c|c|c|}
\hline Name & $\begin{array}{c}\text { Peak position } \\
\mathbf{2 \theta}\end{array}$ & $\begin{array}{c}\text { d- Spacing } \\
\mathbf{2 \theta}\end{array}$ & FWHM & $\begin{array}{c}\text { Crystalline size } \\
(\mathbf{n m})\end{array}$ \\
\hline AERGO & $43^{\circ}$ & 2.10250 & 0.4684 & 19 \\
& & & & \\
CMRGO & $42^{\circ}$ & 2.11684 & 0.4684 & 19 \\
\hline
\end{tabular}

Table 3: XRD Scherrer formula values of AERGO, CMRGO

\subsection{Particle size analysis}

Dynamic light scattering (DLS) is used to know the size of the particle on the material. The pore size distribution focussed in microporous region because the presence of micropores was favourable for greater the better interaction between electrolyte ion and electrode whereas conversely EDLC exhausted the capacity to store the charges if the micropores are allowed to ease the access of electrolyte ions [26].

The particle size analysis of AERGO and CMRGO samples were dispersed in the region of diameter between (454.69 $2222.051 \mathrm{~nm}$ ) at the frequency range of $30.54 \mathrm{~Hz}$. The particle size analysis used to calculate mean, median, , mode and its standard deviations [12]. Dynamic light scattering analysis conducted on the AERGO and CMRGO yielded a nominal diameter of $52 \mathrm{~nm}$ and $0.4 \mathrm{~nm}$ respectively with a standard deviation of 104 and nil $\mathrm{nm}$ respectively. The average diameter was greater than the calculated value from Scherrer's equation for AERGO. Therefore, the synthesised reduced graphene oxide slightly agglomerated [11].

\begin{tabular}{|l|l|l|}
\hline \multicolumn{2}{|c|}{ AERGO } & CMRGO \\
\hline Mean & $51.7 \mathrm{~nm}$ & $0.4 \mathrm{~nm}$ \\
Diameter & & \\
S.D. & $104.0 \mathrm{~nm}$ & - \\
Mode & $12.4 \mathrm{~nm}$ & $0.4 \mathrm{~nm}$ \\
\hline
\end{tabular}

Table 4: Calculation results of AERGO and CMRGO

\subsection{Galvanostatic Charge- Discharge Measurements:}

The charge-discharge curves shown in Figure 7 are almost linear in nature. The capacitive behaviors of AERGO and CMRGO estimated by the galvanostatic charge-discharge performance at constant current density of $2 \mathrm{~A} / \mathrm{g}$ in the potential ranging from 0.0 to $1.2 \mathrm{~V}$ are shown in Figure :7 a-b. It observed that all the curves showed symmetrical triangles with steady variation of slopes, signifying that better EDLC performances. The discharge curves readily concluded the discharging time of the samples. From the discharge curve, the specific capacitance for AERGO and CMRGO were calculated as $149 \mathrm{~F} / \mathrm{g}$ and $151 \mathrm{~F} / \mathrm{g}$ respectively. These outcomes confirm that AERGO and CMRGO were found to have outstanding electrochemical performance.

"The single electrode capacitance was calculated from the galvanostatic discharge process according to the following equation

$$
c=\frac{I \times t}{m \times \Delta V}
$$

Where $I$ is the discharge current (A), $\Delta t$ is the discharge time (s), $\Delta V$ is the voltage change $(\mathrm{V})$ during the discharge process and $m$ is the total mass of the active material" [22]. 
DOI: 10.17148/IARJSET.2021.8852

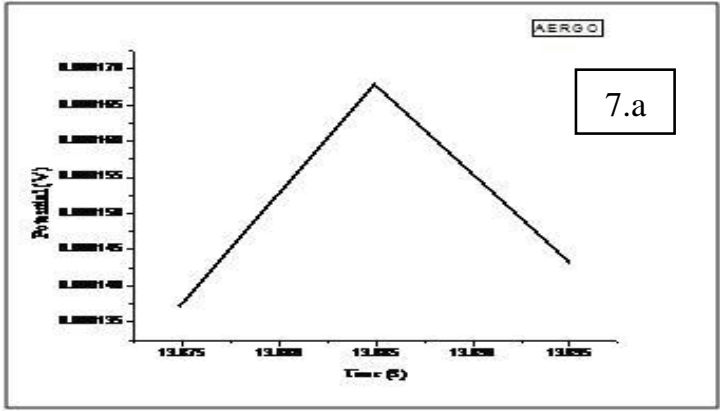

Fig:7.a: Galvanostatic Charge discharge curves of AERGO

\subsection{Impedance Spectroscopy}

It is performed in a frequency range from $100 \mathrm{~Hz}$ to $0.01 \mathrm{~Hz}$ and the results were presented in Figure 8a-b. The Nyquist plot ranging attributed a vertical curve from 100 to $0.01 \mathrm{~Hz}$ and a low faradic charge transfer resistance, representing a closely ideal capacitive performance for AERGO and CMRGO electrode materials. The capacitance as a function of frequency measured from impedance spectroscopy data is shown in Figure 8a-b. Nearly 92.6\% of capacitance retained

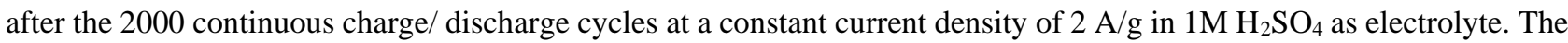
semicircular part at higher frequencies attributed to the electron transfer and its diameter is equal to the Faradic charge transfer resistance (Rct), solution resistance (Rs) is the intersection of the curve at real part $Z^{\prime}$ [28].

Nyquist plot of the CMRGO exhibited a smaller diameter of semicircular in high frequency region than AERGO, signifying that increase in conductivity is attained in the CMRGO system. Simultaneously, the more vertical shape at lower frequencies for CMRGO system indicated a more capacitive behavior of the electrode. Among the tested electrodes, the biomass reduced Graphene oxide electrodes were found to exhibit superior specific conductance.

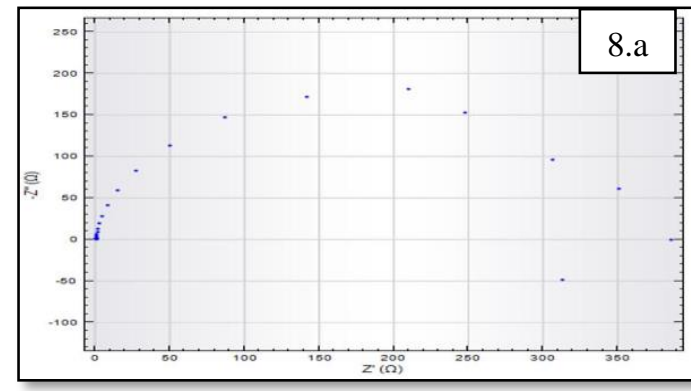

Figure 8.a: Nyquist plot of AERGO

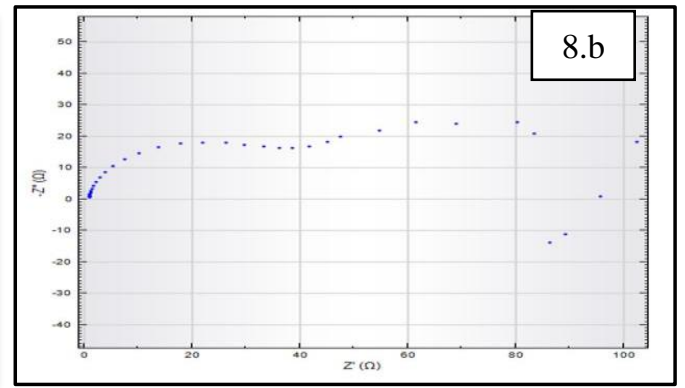

Figure 8.b: Nyquist plot of CMRGO

\section{CONCLUSION:}

Reduction of graphene oxide to reduced graphene oxide using a naturally available Abelmoschus esculentus and Cucumis melo peels are cost effective and environment friendly. The specific capacitance for AERGO and CMRGO were $149 \mathrm{~F} / \mathrm{g}$ and $151 \mathrm{~F} / \mathrm{g}$ respectively. From this study it is concluded that Abelmoschus esculentus and Cucumis melo peel were utilized as the environment- friendly reducing agent and the prepared reduced graphene oxide is used for supercapacitor applications.

\section{ACKNOWLEDGEMENT:}

The authors wish to acknowledge Avinashilingam Institute for Home Science and Higher Education for Women, India, for providing the necessary facilities to carry out the work.

\section{REFERENCES:}

[1]Yumei Ren, Jianmin Zhang, Qun Xu, Zhimin Chen, Daoyuan Yang, Bo Wang and Zheng Jiang (2014) "Biomass-derived three-dimensional porous N-doped carbonaceous aerogel for efficient supercapacitor electrodes", RSC Advances, 4, 23412.

[2] Takur.,S, Karak.,N, Carbon 50 (2012), 5331 - 5339.

[3] Subramanian Ramanathan, Elaiyappillai Elanthamilan, Asir Obadiah, Arulappan Durairaj, Johnson Princy Merlin, Subramanian Ramasundaram,Samuel Vasanthkuamr (2017), “Aloe vera (L.) Burm.f. extract reduced graphene oxide for supercapacitor application”, Journal of Material Science: Materials in Electronics.

[4]Kuila.T, Bose.S, Khanra.p, Mishra.A.K, Kim. N.H., Lee.J.H, carbon 50 (2012), 914-921. 


\title{
International Advanced Research Journal in Science, Engineering and Technology
}

\author{
Vol. 8, Issue 8, August 2021
}

\section{DOI: 10.17148/IARJSET.2021.8852}

[5]Hwei-Jay Chu, Chi-Young, Lee, Nyan- Hwa Tai, "Green reduction of graphene oxide by Hibiscus sabdariffa.L to fabricate flexible graphene electrode" (2014), Eleseveir, 1-8.

[6]Akhavan.O, Kalaee. M, Alavi.Z.S, Ghiasi.S.M.A, Esfandiar, Carbon 50, (2012), 3015-3025.

[7] Wang. Y, Shi.Z, Yin.J, ACS Appl. Mater Interfaces 3 (2011) 1127-1133.

[8]Akhavan.O, Ghaderi.E, Abouei.E, Hatamie.S, Ghasemi.E, Carbon 66(2014), 395-406.

[9] Hwei-Jay Chu, Chi-Young, Lee, Nyan- Hwa Tai, "Green preparation using black soybeans extract for graphene- based porous electrodes and their applications in supercapacitors", Journal of power sources, (2016), 31-39.

[10] Khan.M, A.Al-WArthan,W.Tremel,M.Tahir,M.Siddiqui, Nanoscale Res.Lett. 10 (2015) 1-9.

[11] Katagiri, N., Adachi, N., \& Ota, T. (2014). Preparation and evaluation of ferrite-silica aerogel nanocomposite. Journal of the Ceramic Society of Japan, 122(1421), 29-34.

[12] Toshihide Horikawa, Yukari Ono, J un ichi Hayashi, Katsuhiko Muroyama,(2004), "Influence of surface-active agents on pore characteristics of the generated spherical resorcinol-formaldehyde based carbon aerogels", Carbon 42,2683-2689.

[13] Xi-Lin Wu, Tao Wen, Hong-Li Guo, Shubin Yang, Xiangke Wang and An-Wu Xu (2013) "Biomass-Derived Sponge-like Carbonaceous Hydrogels and Aerogels for Supercapacitors", ACS Nano Vol. xxx ' no. xx '000-000.

[14] Chang.J., Gao.Z., Wang.X., Wu.D., Xu.F., Wang.X., Jiang.K., (2015), “Activated porous Carbon prepared from paulownia flower for high performance supercapacitor electrodes", Electrochim.Acta 212, 839-847.

[15] Abdulhadi H. Al-Marri, Mujeeb Khan, Merajuddin Khan, Syed F. Adil , Abdulrahman Al-Warthan , Hamad Z. Alkhathlan, Wolfgang Tremel , Joselito P. Labis , Mohammed Rafiq H. Siddiqui, Muhammad N. Tahir ,(2015), “Pulicaria glutinosa Extract: A Toolbox to Synthesize Highly Reduced Graphene Oxide-Silver Nanocomposites", Inetrnational Journal of Molecular Science, 16, 1131-1142.

[16] Furong Qin, Kai Zhang, Jie Li, Yanqing Lai, Hai Lu, Wenwen Liu, Fan Yu, Xiaoke Lei, Jing Fang, (2016 ), "Pomegranate rind-derived activated carbon as electrode material for high-performance supercapacitors", Journal of Solid State Electrochem

[17] Sara-Maaria Alatalo (2016) "Hydrothermal Carbonization in the Synthesis of Sustainable Porous Carbon Materials" Acta Universitatis, Lappeenrantaensis 701

[18] Wang, G., Zhang, L., \& Zhang, J. (2012). A review of electrode materials for electrochemical supercapacitors. Chem. Soc. Rev., 41(2), 797-828. [19] Wang, R., Wang, P., Yan, X., Lang, J., Peng, C., \& Xue, Q. (2012). Promising Porous Carbon Derived from Celtuce Leaves with Outstanding Supercapacitance and CO2 Capture Performance. ACS Applied Materials \& Interfaces, 4(11), 5800-5806.

[20] Joji Reddy.B, Vickraman. P,Simon Justin. A, (2018), "Moringa oleifera Leaf Extract Mediated Reduced Graphene Oxide/ $\alpha$-Ni(OH)2 Nanocomposite for Asymmetric Supercapacitors", Brazilian Journal of Physics.

[21] Vijayakumary.P, "Biomass Management for Fodder and Energy".

[22] Ruili Liu, Li Wan, Shaoqing Liu, Lixia Pan, Dongqing Wu, Dongyuan Zhao,(2014), “An Interface-Induced Co-Assembly Approach Towards Ordered Mesoporous Carbon/Graphene Aerogel for High-Performance Supercapacitors", Advanced Functional Materials, 1-8.

[23] Sourav Sadhukhan, Tapas Kumar Ghosh, Dipak Rana, "Studies on synthesis of reduced graphene oxide (RGO) via green route and its electrical property", (2016).

[24] Chaitra K , Nagaraju N. , Kathyayini Nagaraju, (2015), "Nanocomposite of hexagonal b-Ni(OH)2/multiwalled carbon nanotubes as high performance electrode for hybrid supercapacitors", Materials Chemistry and Physics, 1-10.

[25] Kalaivani.T, Tharani.S, Prithiba.A,(2020), "Biomass Derived Reduced Graphene oxide from Abelmoschus esculentus and Cucumis melo peels as electrode material for High Performance Supercapacitors", First International Conference on Frontiers in Chemical Sciences (ICFCS),page no.69.

[26] Xin Liu, Pingping Song, Jiahui Hou, Bo Wang, Feng Xu, Xueming Zhang (2018), "Revealing the Dynamic Formation Process and Mechanism of Hollow Carbon Spheres: from Bowl to Sphere's Shape”, ACS Publications, 1-31.

[27] Syed Nasimul Alam, Nidhi Sharma, Lailesh Kumar,(2017), "Synthesis of Graphene Oxide (GO) by Modified Hummers Method and Its Thermal Reduction to Obtain Reduced Graphene Oxide (rGO)", Scientific Research.

[28] Rutao Wang, Peiyu Wang, Xingbin Yan, Junwei Lang, Chao Peng, Qunji Xue, (2012), "Promising porous carbon derived from celtuce leaves with outstanding supercapacitance and $\mathrm{CO}_{2}$ capture performance", ACS Publications.

[29] Chao Peng, Xing-bin Yan, Ru-tao Wang, Junwei Lang, Yu-jing Ou, Qun-ji Xue. "Promising activated carbons derived from waste tealeaves and their application in high performance supercapacitors electrodes" , Electrochimica Acta, 2013 